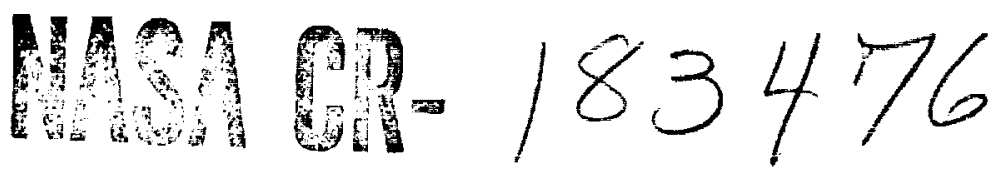

DESIGN OF A HIGH-GAIN LASER DIODE-ARRAY PUMPED Nd:YAG ALTERNATING PRECESSIVE SLAB AMPLIFIER (APS-AMPLIFIER)

\author{
D. Barry Coyle \\ The American University \\ Department of Physics \\ Washington, DC 20016 USA
}

This work funded by NASA-GSFC Contract \#NSG-50333

and performed at

NASA - Goddard Space Flight Center

Code 726, Photonics Branch

Greenbelt, MD 20771 USA

\begin{abstract}
In the design of space-qualifiable laser systems for ranging and altimetry, such as NASA's Geodynamic Laser Ranging System (GLRS), the transmitter must be kept small, powerful yet efficient, and must consist of as few components as possible. This paper focuses on a novel preamplifier design that requires no external beam-steering optics, yielding a compact component with simple allgnment procedures. The gains achleved are comparable to multipass zigzag amplifiers using two or more sets of external optics for extra passes through the amplifying medium.
\end{abstract}


Introduction

In the design of diode-pumped laser system candidates for space-based laser ranging and altimetry projects such as the Geoscience Laser Ranging System every vable idea for decreasing size, weight, and energy requirements of the laser systems is sought. The fundamental pulsewidths are currently required to be less than $100 \mathrm{ps}$ at a 40 pulse per second repetition rate due to the resolution and integration time requirements of proposed streak tube data-taking systems[1]. This paper reports on a unique preamplifier design which satisfies the above requirements, and is suitable as the first amplifier stage following a laser of $1 \mathrm{~mJ}$ output.

Of the laser amplifier geometries studied, the zigzag amplifiers display the best over-all extraction efficiencies and temperature stabilities required for space. A multipass scheme is commonly used to achieve high gain. However, extra optics and space to fold the beam is required. An example of a high gain, slab amplifier is the $62 \mathrm{~dB}$ multipass zigzag amplifier built by Kane, Kozlovsky, and Byer[2] in 1986 employing external optics for four complete round trips. Our application requires an amplifier of smaller scale, with attendant smaller gain and temperature stabllity features. Chan[3] reports on a zigzag amplifier employing only one transmissive face for both the pulse's entry and exit. The design is intended for space-based altimetry but also requires extra hardware to increase the gain length. By studying pros and cons of zigzag geometries we 
have developed a similar design that keeps the amplified pulse in the gain medium as long as possible but requires no additional hardware. A method for designing an Alternating Precessive Slab (APS) Amplifier is presented.

\section{General Parameters}

A rectangular path was chosen by shortening a side of a square. By sending the beam in off-diagonal, shown in Fig. 1, a $32 \mathrm{~cm}$ long multipass trace is produced inside of a Nd:YAG slab. All angles of incidence in the medium are $45^{\circ}$ and use Total Internal Reflection (TIR) properties. Each line inside the slab represents the center of a gaussian beam which has a cross sectional area of

$$
A=\pi \omega^{2},
$$

where $\omega$ is the collimated beam waist entering the medium from a short-pulse (100 ps) laser. For minimum overlap of the pulse in the gain medium, we impose the condition

$$
d \geq 2 \omega \cos \left(45^{\circ}\right),
$$

where $d$ represents the distance between beam centers on any of the four reflective faces. Equation (2) must be followed in order to keep the internally reflecting pulse from occupying any of the neighboring beam's volume. If the

distances between neighboring, parallel lines represent the beam centers and are all a distance of exactly $2 \omega$ apart, then in two dimensions the total area of the slab is swept through twice before the pulse leaves the cavity. Given the above geometrical parameters, it is possible to design an amplifier for our needs. 
In the absence of divergence, the entry and exit faces would be the same diameter as the input beam diameter for maximum extraction efficiency. However, it is necessary to account for beam spreading within the medium. For a cylindrical rod amplifier, Degnan[4] identifies a trade-off between amplifier extraction efficiency and beam quality for rod amplifiers. Phase or amplitude fluctuations can be triggered by diffraction effects caused by the aperture-radius to beam-waist ratio. Any one of these fluctuations can lead to self-focusing in the rod at high pulse powers.

The thickness of the slab determines the entrance and exit aperture diameters. By choosing a thickness of $t=0.21 \mathrm{~cm}$, our aperture radius is $r=t / 2=0.105$ $\mathrm{cm}$. Since the spatial pulse shape will grow upon amplification, the desired ratio should be slightly greater than unity, about 1.05 , where

$$
\left(\frac{t}{2}\right) \frac{1}{\omega}=1.05 .
$$

Then $t=0.21 \mathrm{~cm}$ and $\omega=0.20 \mathrm{~cm}$. To allow for this extra "room" between neighboring pulses and the entry/exit apertures, equation (2) is replaced with

$$
d \geq t \cos \left(45^{\circ}\right),
$$

where $\mathrm{d}=0.297 \mathrm{~cm}$.

The slab, which is a $0.8 \%$ doped Nd:YAG and lases at $1064 \mathrm{~nm}$, is pumped at $809 \mathrm{~nm}$ from the four polished sides, A, A(2), and both B's, with linear laser diode arrays. See Fig. 1 and 2. By matching the diode array widths to those of the pumping faces of the slab amplifier, symmetrical temperature distributions 
across the $x-y$ plane are formed, a common characteristic of zigzag geometries. The temperature/energy analysis will be presented later. The coatings will be anti-reflective (AR) for $809 \mathrm{~nm}$ on the pumping faces, and AR for $1064 \mathrm{~nm}$ on the entry/exit faces. An $8 \times 8 \mathrm{~cm}$ square model was assembled with silver-coated microscope slides to represent the sides of the rectangle. The path in the simulated crystal was not destroyed when the opposing optical faces were non-parallel by about $1.0^{\circ}$. With the opposing faces of the crystal not exactly parallel, parasitic oscillations in the $x-y$ plane can be reduced. Furthermore, the beam retained the same gaussian shape it possessed before entering the test cavity. The top and bottom faces will have a frosted finish in order to be non-reflective and supply a good heat sink. Fig. 3. A brief analysis of parasitics is covered later.

\section{Example Design}

Due to the chosen geometry, the number of paths, $N$, in the $x$ and $y$ directions are equal. The location of the exit face, holding the position of the entry face constant, alternates between opposite "corners" for odd and even N's. The length of each side is calculated from Table 1.

The emitting facets of the chosen diodes are $10 \mathrm{~mm}$ wide by $0.3 \mathrm{~mm}$ high. The pump light emerging from these apertures is divergent by $\theta_{(x y)}=10^{\circ}$ and $\theta_{(z)}=$ $35^{\circ}$ respectively, with the xy plane being the same as that of the slab in Fig. 2. 
From Table 1, it is found that $\mathrm{N}=9$ allows each pump face to have enough area for the $\theta_{(x y)}$ diverging light to couple into the slab. This forces each of the pump faces to be slightly wider than $1 \mathrm{~cm}$. Fig. 3 shows the parameters for an $\mathrm{N}=8$ slab as an example. Coupling of the $\theta_{(z)}$ light is accomplished by using cylindrical glass rods $3 \mathrm{~mm}$ in diameter, (Suprasil from Heraeus Amersil), as collimating lenses. These rods are fire-polished fused silica with a transmission of over $95 \%$ at $\lambda=809 \mathrm{~nm}$. By varying the diode to rod distance, a $2 \mathrm{~mm}$ thick beam can be maintained for more than 4 centimeters. A $3 \mathrm{~mm}$ diameter rod seemed to work the best in our tests. Other techniques were considered, including fiber optic coupling. In the first version of the amplifier, uncoated rods will be used, however AR coatings on the glass rods can reduce pumping losses even more.

The total slab volume, after finding the even or odd parameters, is found by

$$
V=\left(A B+\frac{d}{2}\left(A(2)+\frac{d}{2}\right)\right) t
$$

The volume determines the pumping requirements of the diodes. Using $d=$ $0.297 \mathrm{~cm}$ and the data found in Table 1 for $\mathrm{N}=9 \mathrm{in}$ equ. (5) a volume of $\mathrm{V}=$ $0.333 \mathrm{~cm}^{3}$ is computed. The path length inside the medium, or the gain-path length $L_{p}$, can be found by summing the paths in the slab, or

$$
L_{P}=\sum L_{x}+\sum L_{y}
$$

where $L_{x}$ and $L_{y}$ represent the distance travelled by the pulse in the $x$ and $y$ directions. Recalling that the beam centers are a distance $t$ apart, then 


$$
L_{P}=2\left[\left(N-\frac{1}{2}\right)+\sum_{i=1}^{N-1}(i t)\right]
$$

is the total gain-length inside the crystal. Following the injected pulse's path in Fig. 2, we see in both the vertical and horizontal axes, traces $1-8$ and $\mathrm{a}-\mathrm{h}$ respectively, the order of the paths alternate from side to side. Due to this alternation and apparent precession of rectangular paths, the design is referred to as the Alternating Precessive Slab Amplifier, or the APS Amplifier.

\section{Numerical Analysis of an $\mathrm{N}=9$ APS Amplifier}

Degnan[5] notes that the saturation energy density for sub-nanosecond pulsewidths is

$$
D_{s a t}=\frac{h v}{2 \sigma L\left(v-v_{0}\right)}
$$

where $\sigma$ is the stimulated emission cross section, $v$ is the amplifying pulse center frequency, $v_{0}$ is the resonant frequency of the gain medium and $L\left(v-v_{0}\right)$ is the Lorentzian profile given by

$$
\mathcal{L}\left(v-v_{0}\right)=\frac{1}{1-\left(\frac{v-v_{0}}{\gamma_{a b}}\right)^{2}} .
$$

The lifetime broadening of the upper and lower Nd lasing levels is $\gamma_{a b}$ or

$$
\gamma_{a b}=\frac{1}{2}\left(\gamma_{a}+\gamma_{b}\right)
$$

where $\gamma_{a}$ and $\gamma_{b}$ are the upper and lower transition rates respectively. The injection of a 100 ps pulse from a diode-pumped, injection-locked, Nd:YAG ring laser is assumed and $v=v_{0}$. The saturation energy density is then 


$$
D_{\text {sat }}=\frac{h v_{l}}{2 \sigma},
$$

where $v_{1}=2.82 \times 10^{14} \mathrm{~Hz}$ is the Nd:YAG lasing frequency at $1064 \mathrm{~nm}$.

The Nd ion density of the Nd:YAG slab is $N_{1}=1.38 \times 10^{20}$ ions $/ \mathrm{cm}^{3}$. The total pump energy absorbed in the ${ }^{4} \mathrm{~F}_{3 / 2} \mathrm{Nd}$ upper lasing level is then

$$
E_{d}=\eta_{1} \eta_{2} \eta_{3} \eta_{4} \eta p f_{a} T_{p} 4
$$

where $\eta_{1}=0.9$ is the optical coupling efficiency into the slab, $\eta_{2}=0.95$ is the absorption efficiency, $\eta_{3}=0.76$ is the color efficiency or the ratio of the pump and laser wavelengths. The fluorescence efficiency, $\eta_{4}$, is calculated by

$$
\eta_{4}=\frac{\tau_{2}}{T_{p}}\left[1-\exp \left(-\frac{T_{p}}{\tau_{2}}\right)\right]=0.76 .
$$

It is the fraction of pump energy absorbed in the medium that is integrated and stored[6]. $T_{p}=200 \mu \mathrm{sec}$ is the pump pulsewidth and $\tau_{2}=230 \mu \mathrm{sec}$ is the spontaneous fluorescence lifetime. The Boltzman constant $f_{a}=0.41$ is the fraction of atoms in the ${ }^{4} \mathrm{~F}_{3 / 2}$ level that are actively involved in the stimulated emission process. The power per diode array is $p=120 \mathrm{~W}$ and there are four of them. The diode arrays are from Spectra Diode Labs and are 2-bar stacks with each bar emitting $12 \mathrm{~mJ}$. From equ. (12) and (13), $18.7 \mathrm{~mJ}$ is stored in the upper lasing level in the slab.

A Fortran program has been developed to simulate the amplifier during operation.[7] The graphical output in Fig. 4 shows that after pumping and 
prior to pulse extraction, the energy distribution across the $x-y$ plane of the slab possesses no dangerous hot spots and is fairly symmetric. The plot shown is for an $\mathrm{N}=9$ crystal where $\mathrm{t}=0.21 \mathrm{~cm}, \mathrm{~A}=1.48 \mathrm{~cm}$ and $\mathrm{A}(2)=\mathrm{B}=1.19 \mathrm{~cm}$.

The program begins by pumping the APS slab from the 4 sides with 4 collimated pump beams which are $1 \mathrm{~cm}$ wide by $0.21 \mathrm{~cm}$ high. The slab is divided into a 40 x 40 two-dimensional array of 'pods' whose grid axes follow the pump directions. Each pod is then assigned the resultant energy value stored in its volume. The pumping calculation begins with just one of the four diode arrays. By calculating the wavefront intensity, I, as it propagates through the slab, the absorption equation is

$$
I_{n}=I_{n-1} \exp \left(-N_{1} \sigma_{a b s} \Delta X\right),
$$

where $N_{i}$ is the Nd ion density, $\sigma_{a b s}$ is the absorption cross section, and $\Delta X$ is the pod thickness. The subtraction of each successive $I_{n}$ from the previous $I_{n-1}$ before and after each pod yields the change in Intensity, $\Delta I$, per pod. The energy deposited per pod is obtained by multiplying the pulsewidth, $T_{p}=200$ $\mu s$, and the pod's cross sectional area. Applying the efficiencies used in equ. (14) the energy per pod stored for stimulated emission is found. A surface plot as a result of only a single diode array is shown in Fig. 5 . This procedure is repeated for each of the 4 diode arrays by superposition and the total energy per pod is obtained. See Fig. 5.

For the actual amplification simulation, the pods are then combined into larger units we refer to as cells. These cells are laid out in a grid essentially $45^{\circ}$ 
different than the pod grid. This helps to simplify gain calculations for the amplifying pulse and to account for the slab's double-pass feature. Using Verdeyen[8] the small signal gain, $G_{0}$, per cell is calculated, which is now aligned with the internal beam trace using

$$
G_{0}=\exp \left(\sigma N_{2} L_{p}\right) .
$$

From Frantz and Nodvik[9] the resulting energy density is found to be

$$
D_{\text {out }}=D_{\text {sat }} \ln \left[1+\left[\exp \left(\frac{D_{\text {in }}}{D_{\text {sat }}}\right)-1\right] G_{0}\right],
$$

where $D_{\text {sat }}=h v / 2 \sigma$ is the saturation energy density. By using the output energy density, $D_{\text {out }}$, of the previous cell as the input energy density, $D_{i n}$, of the present cell, the change in energy, $\Delta \mathrm{E}$, can be found per cell, or

$$
\Delta E_{\text {cell }}=\left(D_{\text {out }}-D_{\text {in }}\right) A
$$

with A being the beam cross section. Since there is essentially a 1:1 ratio of stimulated relaxations to emitted photons, we can say that the change in the Nd inversion density is

$$
\Delta N_{2}=\frac{\Delta E}{E_{p h}},
$$

where $E_{p h}$ is the energy per $1064 \mathrm{~nm}$ photon. A new $\mathrm{N}_{2}$ for the second pass can be found for the cell in order to find the new $G_{0}$ by simply subtracting $\Delta N_{2}$ from the $\mathrm{N}_{2}$ prior to the first pass. Eventually, the pulse travels the entire internal path and all the $\Delta E$ 's are all summed to find the final $E_{\text {out }}$.

It was discovered that a standard $1.0 \% \mathrm{Nd}$ :YAG slab would not be as efficient as one with a lower doping density. The Fortran code modeled slabs with 
densities of $0.5 \% \mathrm{Nd}$ through $1.1 \%$. Most of the energy is absorbed near the pump face. Fig. 5 shows the result of a 1.0\% slab pumped with 1 diode array. The best results were obtained with $0.8 \%$ concentration. When pumping with a single array, the energy deposited in the center pods is about $25 \%$ of those at the pump face. After pumping from 4 sides the energy stored in the center will about equal that of the face as pumped with a single diode. The addition of 4 sources allows for a symmetric and relattvely smooth energy distribution. Fig. 4.

For our $\mathrm{N}=9$ slab, we simulated the APS Amp with an input pulse of energy $E_{\text {in }}$ $=1.0 \mathrm{~mJ}$, at $\lambda=1064 \mathrm{~nm}$ and a pulsewidth of $\mathrm{T}=100 \mathrm{ps}$. Four SDL diode array pump sources results in a total pump power of $480 \mathrm{~W}$ with $96 \mathrm{~mJ}$ of energy. Using the above data for an $N=9$ slab, an output energy of $E_{\text {out }}=15.1$ $\mathrm{mJ}$ is calculated. Our extraction efficiency, $E_{\mathfrak{l}}$, is found by

$$
E_{f}=\left(\frac{\left(E_{\text {out }}-E_{\text {in }}\right)}{E_{d}}\right) 100=68.1 \%,
$$

where $E_{d}=18.0 \mathrm{~mJ}$ is the total stored energy in the slab.

In high gain media, such as pulsed nitrogen lasers, cavity mirrors for optical feedback are often not required. A coherent beam is produced by cavities supporting only single passes in the laser material. These are called Amplified Spontaneous Emission or ASE Lasers. In lasing media such as Nd:YAG where the gain per-unit-length is not so high, ASE is usually not a factor unless other ASE supporting conditions exist. Due to the relatively long gain path length 
within the APS crystal, Amplified Spontaneous Emission might prove to be a problem. Siegman discusses these conditions for a sample laser rod of diameter 2a and length $\mathrm{L}_{\mathrm{p}}$. A worst case scenario for the APS Amplifier can be studied by applying Siegman's methods to a $32 \mathrm{~cm} \mathrm{Nd:YAG} \mathrm{rod,} 0.21 \mathrm{~cm}$ in diameter. This is the equivalent 'unfolded' gain path within the slab. Let $\mathrm{W}_{12}$ be the stimulated transition rate of Nd inversions due to a travelling ASE pulse of intensity I and $\gamma_{\mathrm{ab}}$ be the natural spontaneous decay rate of the inverted $\mathrm{Nd}$ atoms.[10] The ratio of $W_{12}$ and $\gamma_{a b}$ describes how the ASE effects the natural inversion lifetime. From Siegman we have,

$$
W_{12}=\sigma \frac{I}{h v},
$$

where I is the intensity of the ASE induced pulse. The natural inversion decay rate is

$$
\gamma_{a b}=\frac{4 \sigma L_{p}^{2} I}{h v a^{2}} \exp \left(-2 \alpha_{m} L_{p}\right)
$$

Let $2 \alpha_{m}=N_{2} \sigma$ be the amplification coefficient in the rod where $\mathrm{N}_{2}$ is the average inversion density in the slab. Dividing equ. (20) by equ. (21) yields

$$
\frac{W_{12}}{\gamma_{a b}}=\left(\frac{a}{2 L_{p}}\right)^{2} \exp \left(2 \alpha_{m} L_{p}\right) .
$$

Plugging the necessary values of equ. (16) and $\sigma=9.0 \times 10^{-19} \mathrm{~cm}^{2}[11]$ into equ. (22) ylelds a ratio of 0.0022 . Thus, only $0.22 \%$ of the natural spontaneous decay is effected by ASE at each end of the 'unfolded' rod. There are many published values of the stimulated emission cross section, $\sigma$. The value presented here, which seems a little high, was chosen for these calculations to 
allow for a maximum ASE effect. Thus, a reduction in inversions of $0.22 \%$ is the worst case scenario. The overlapping nature of the gain path was not included. It is true that ASE can arise from any direction within the gain medium where the path is long or the gain is high. Other directional parasitics were not of concern since there are no other relatively long paths foreseen within the slab. The optical faces are designed to be slightly off-parallel by less than $1^{\circ}$ to reduce any possible Fabry-Perot cavitles. This precaution need not be of concern anyway since there are no high reflective coatings at $1064 \mathrm{~nm}$.

\section{Conclusion}

A very efficient amplifier has been designed in order to maximize extraction efficiency while keeping the number of external components to a minimum, with the only critical external beam requirements being the input beam diameter and its energy. At the time of this writing the crystal has been ordered from Lightning Optical in Florida and all the mounting components have been machined and assembled. We have already 4 diode arrays at 50 Watts each to perform tests untll the 120 Watt arrays are received. Fig. 6 shows the top view of an assembled APS Amplifier with the J-mount SDL diodes. After injecting a $100 \mathrm{ps}, 1.0 \mathrm{~mJ}$ pulse, a $15.1 \mathrm{~mJ}$ output was calculated with minimum ASE effects. This results in an amplifier with 11.8 $\mathrm{dB}$ net gain and a $68.1 \%$ extraction efficiency.

The assembled size measures 3 inches square and 2 inches high with the crystal being mounted on the top and bottom large faces. The APS Amplifier is 
a very compact design. The initial test assembly will be water cooled with plans to adapt to a large thermo-electric cooler. At $40 \mathrm{~Hz}$ and $200 \mu \mathrm{s}$ pump pulses, the heat dumped by a $0.8 \%$ duty cycle will not be difficult to remove from the apparatus.

This research is part of an ongoing grant (\#NSG-50333) from the National Aeronautics and Space Administration through the American University, Department of Physics, and is performed at the Goddard Space Flight Center, Code 726 Photonics Branch, in Greenbelt, MD.

\section{Acknowledgement}

The author would like to thank Tammy Bertram for her computer abllities and her patience and Dr. Kay for his advice. 
Figure Captions

1. The geometrical parameters for an $N=8$ APS Amplifler. The z-axis projects out of the paper. For an $\mathbf{N}=9$ design, keeping the entry face on the right, the exit aperture will switch to the bottom.

2. By tracing the beam path through the slab, the volume is symmetrically swept through twice. The diode arrays are $1 \mathrm{~cm}$ in length.

3. Coating and polishing specifications for the crystal. Slab is mounted on the top and bottom faces which are also roughened for parasitic dampening.

4. Energy distribution plot of the APS Amplifier crystal with only 1 diode array. Note: this is for a $1.0 \%$ Nd:YAG slab. The doping was lowered to $0.8 \%$ in order to provide a better center-to-face ratio of deposited energy.

5. Energy distribution plot of the APS Amplifier crystal after pumping and prior to pulse injection. The top and middle right corners have zero stored energy since they are the entry and exit faces.

6. The top view of an assembled APS Amplifier with $4 \mathrm{~J}$-type SDL arrays. Dimensions are roughly 3 " $\times 3 "$ in size. 
Table 1

\begin{tabular}{|c|c|c|}
\hline Face & $\mathrm{N}=$ Even & $\mathrm{N}=$ Odd \\
\hline (A) & $A=\frac{N_{\text {even }}}{2} d$ & $A=\left(\frac{N_{\text {odd }}}{2}+0.5\right) d$ \\
(A2) & $A(2)=\left(\frac{N_{\text {even }}}{2}-1\right) d$ & $A(2)=\left(\frac{N_{\text {odd }}}{2}-0.5\right) d$ \\
(B) & $B=A$ & $B=A(2)$ \\
\hline
\end{tabular}




\section{References}

[1]. S.C. Cohen, J.J. Degnan, III, J.L. Bufton, J.B. Garvin, J.B. Abshire, "Geoscience Laser Ranging System", IEEE Trans. Geosci. Remote Sensing, vol GE-25, no. 5, pp. 581-592, 1987.

[2]. T.J. Kane, W.J. Kozlovsky, R.L.Byer, "62-dB-gain Multiple-pass Slab Geometry Nd:YAG Amplifier", Opt. Lett. vol. 11, pp. 216-218, 1986.

[3]. K. Chan, "Multiple-pass Laser-diode-pumped Nd:YAG Amplifier: Design", Appl. Opt., vol. 26, no. 16, pp. 3177-3179, 1987.

[4]. J.J. Degnan, "Physical processes affecting the performance of high power, frequency-doubled, short pulse laser systems: Analysis, simulation, and experiment", Ph.D. dissertation, Univ. Maryland, College Park, May 1979, pp. 50 .

[5]. J.J. Degnan, "Physical processes affecting the performance of high power, frequency-doubled, short pulse laser systems: Analysis, simulation, and experiment", Ph.D. dissertation, Univ. Maryland, College Park, May 1979, pp. 50 .

[6]. Norman P. Barnes, Mark E. Storm, Patricia L. Cross, Milton W. Skolaut, JR. "Efficiency of Nd Laser Materials with Laser Diode Pumping", IEEE FQE, Mos. 26, No. 3, pp. 558-569, 1990!

[7]. Tammy L. Bertram, D. Barry Coyle, to be published.

[8]. J.T. Verdeyen, Laser Electronics, New Jersey: Prentice-Hall, 1981.

[9]. L.M. Frantz and J.S. Nodvick, "Theory of Pulse Propagation in a Laser Amplifier," J. Appl. Phys. vol. 34, no. 8, pp. 2346-2349, 1963.

[10]. Anthony E. Siegman, Lasers, University Science Books, 1986.

[1 1]. Peter W. Milonni, Joseph H. Eberly, Lasers, John Wiley and Sons, 1988. 


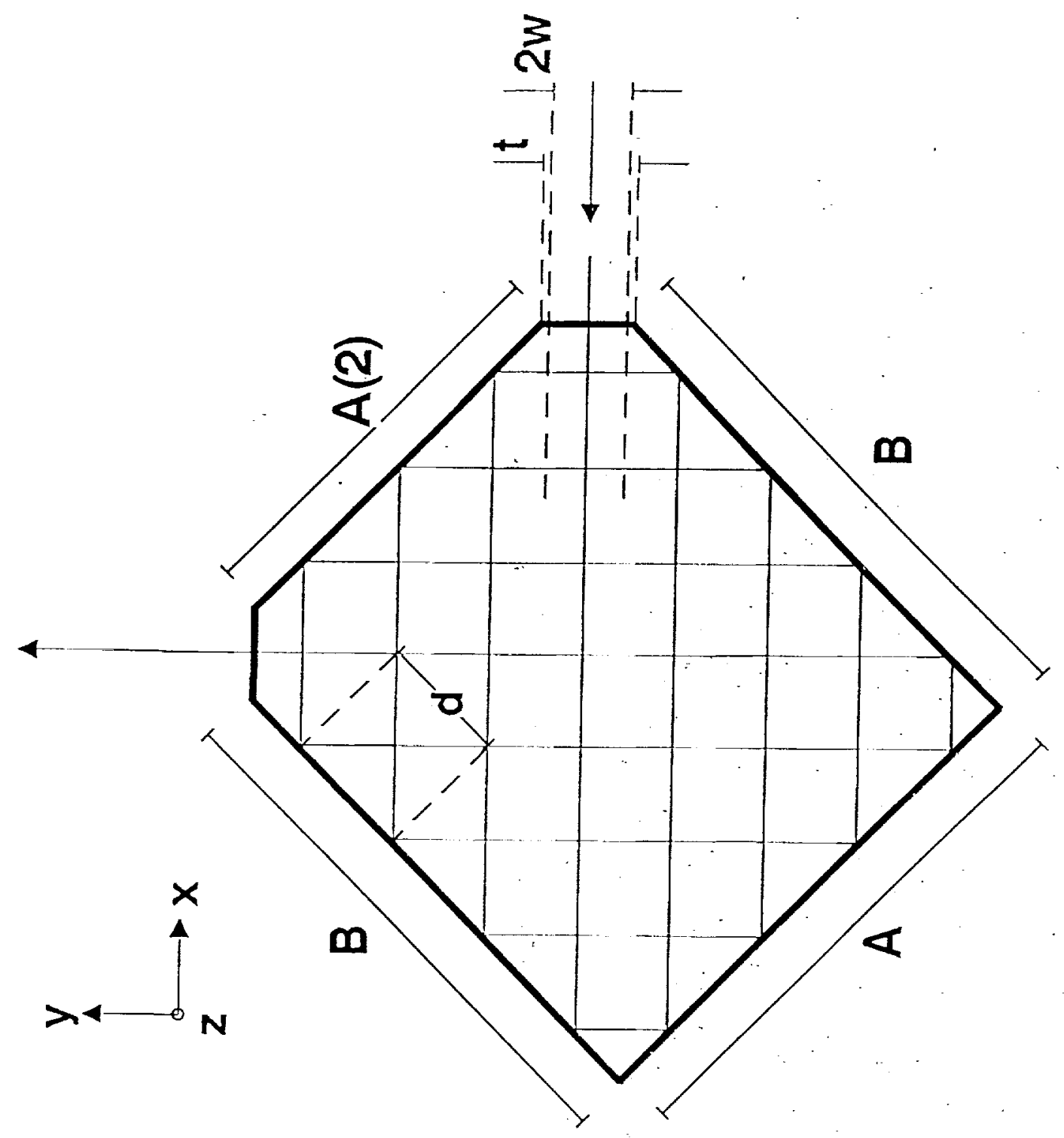




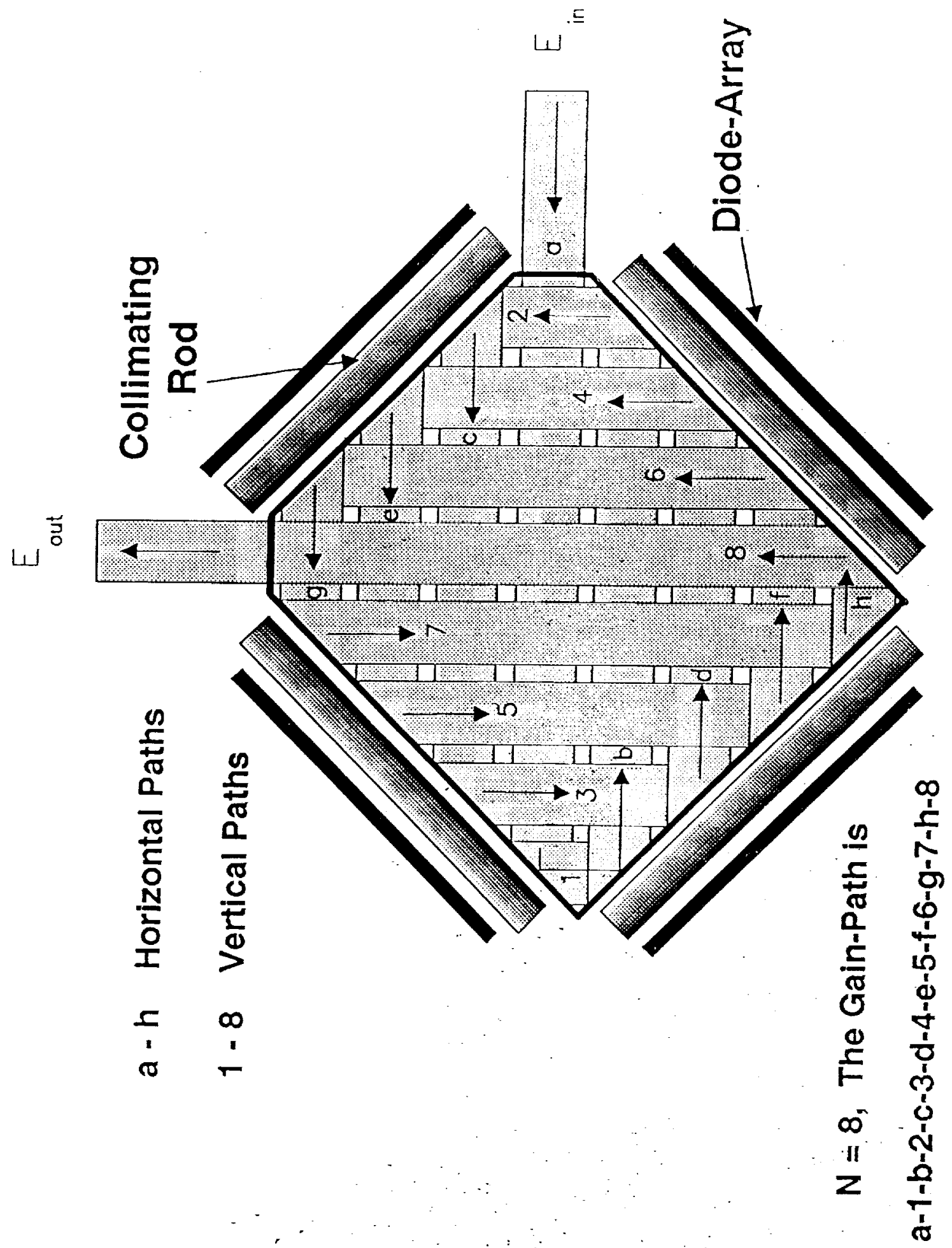




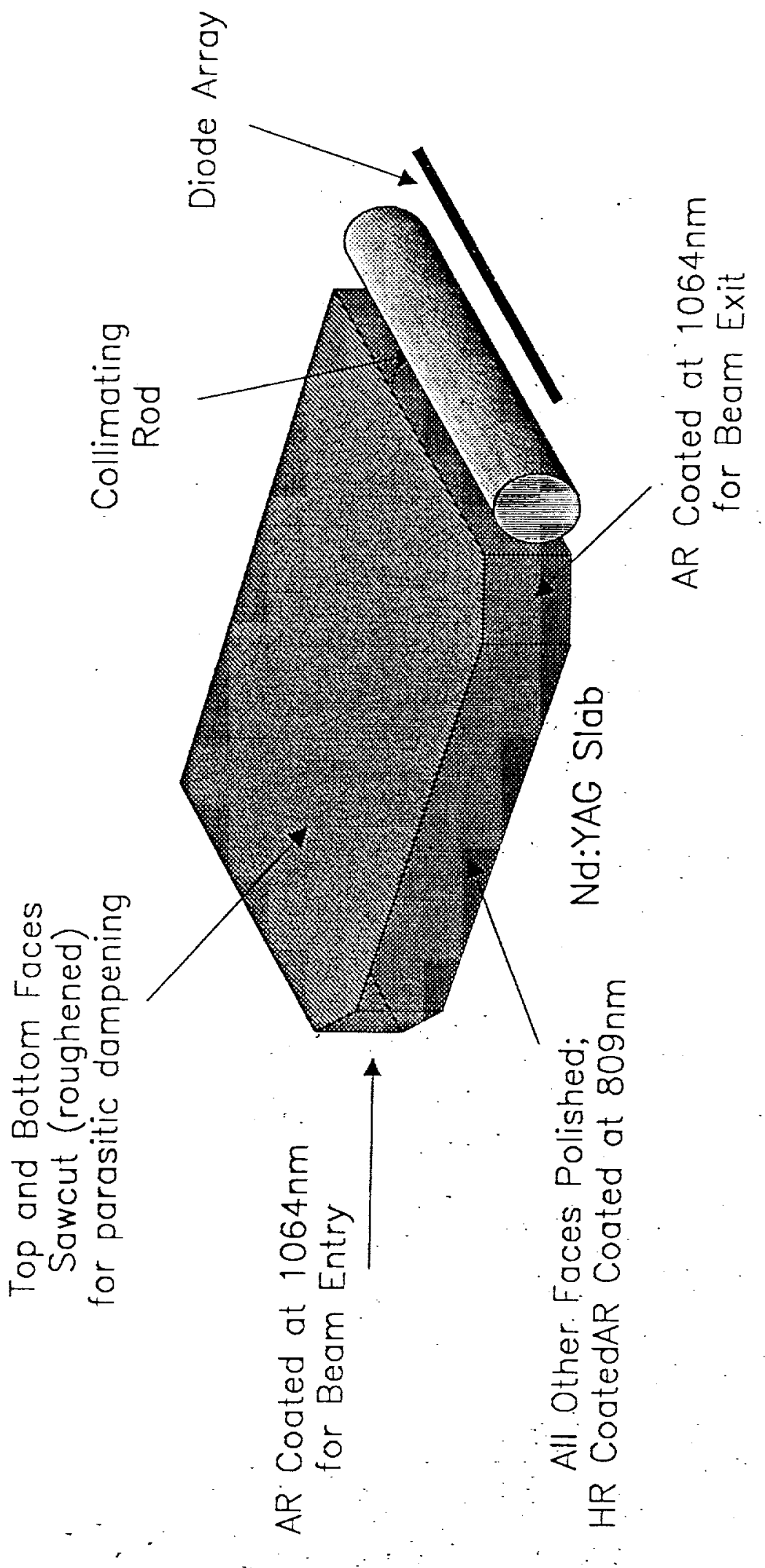




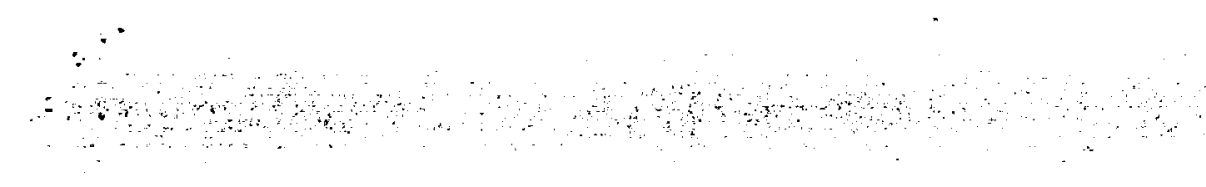

ENERGY DISTRIBUTION

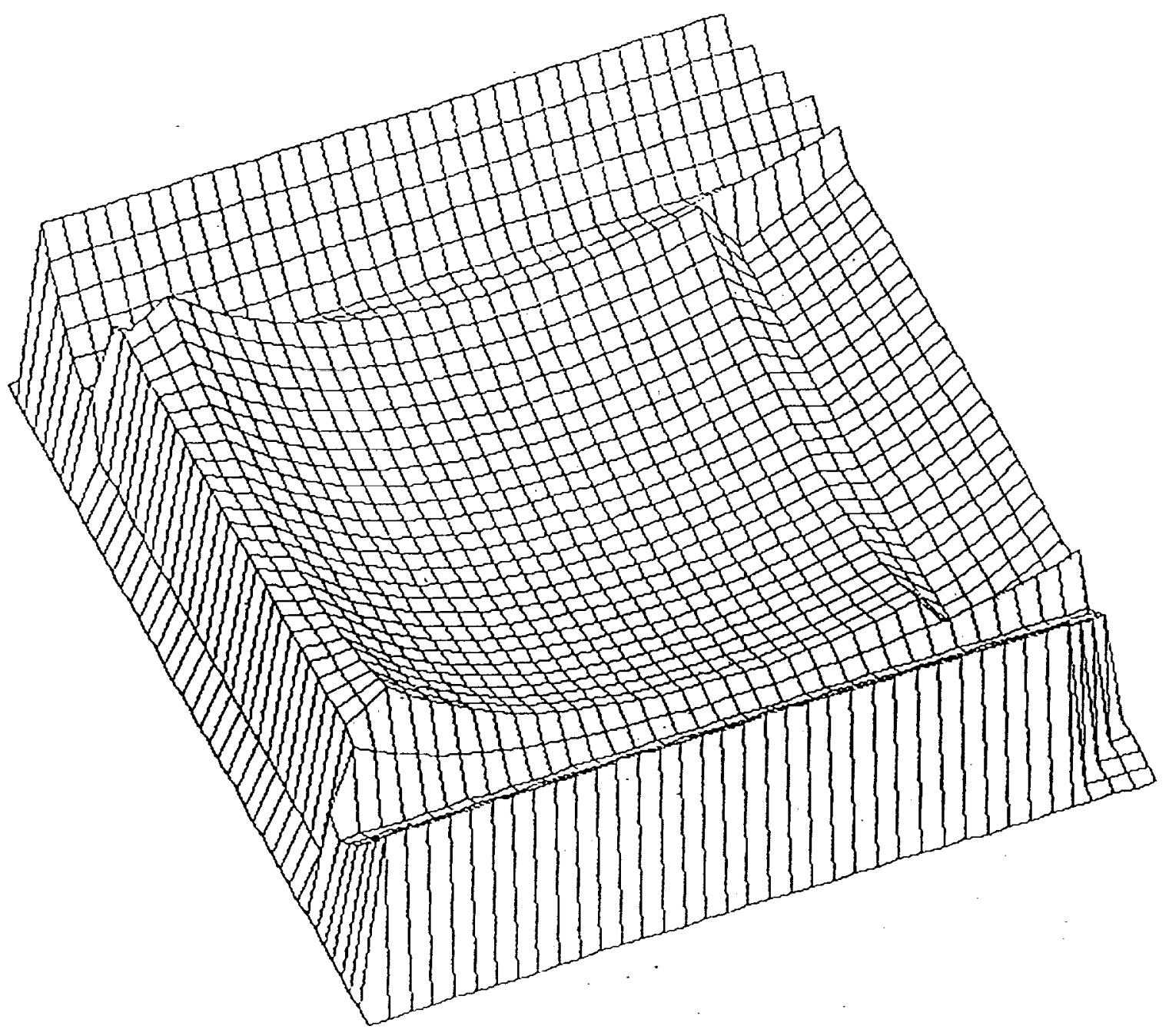




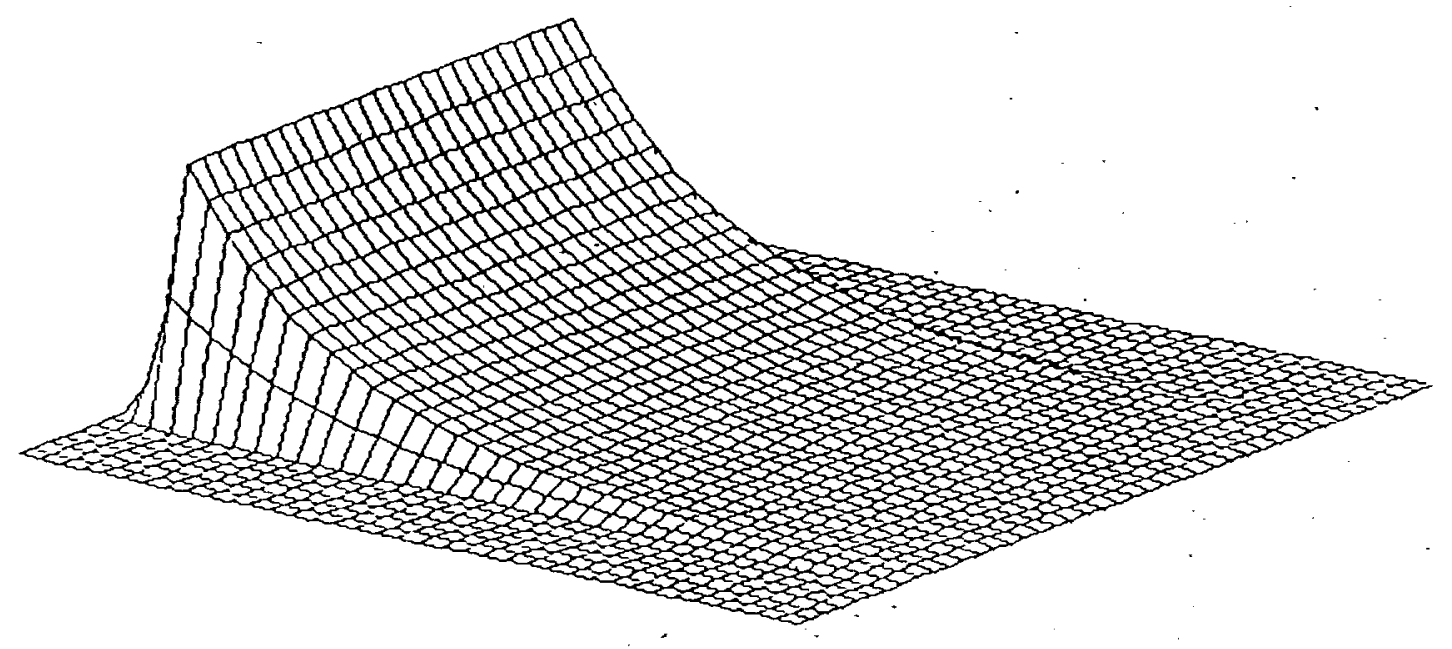




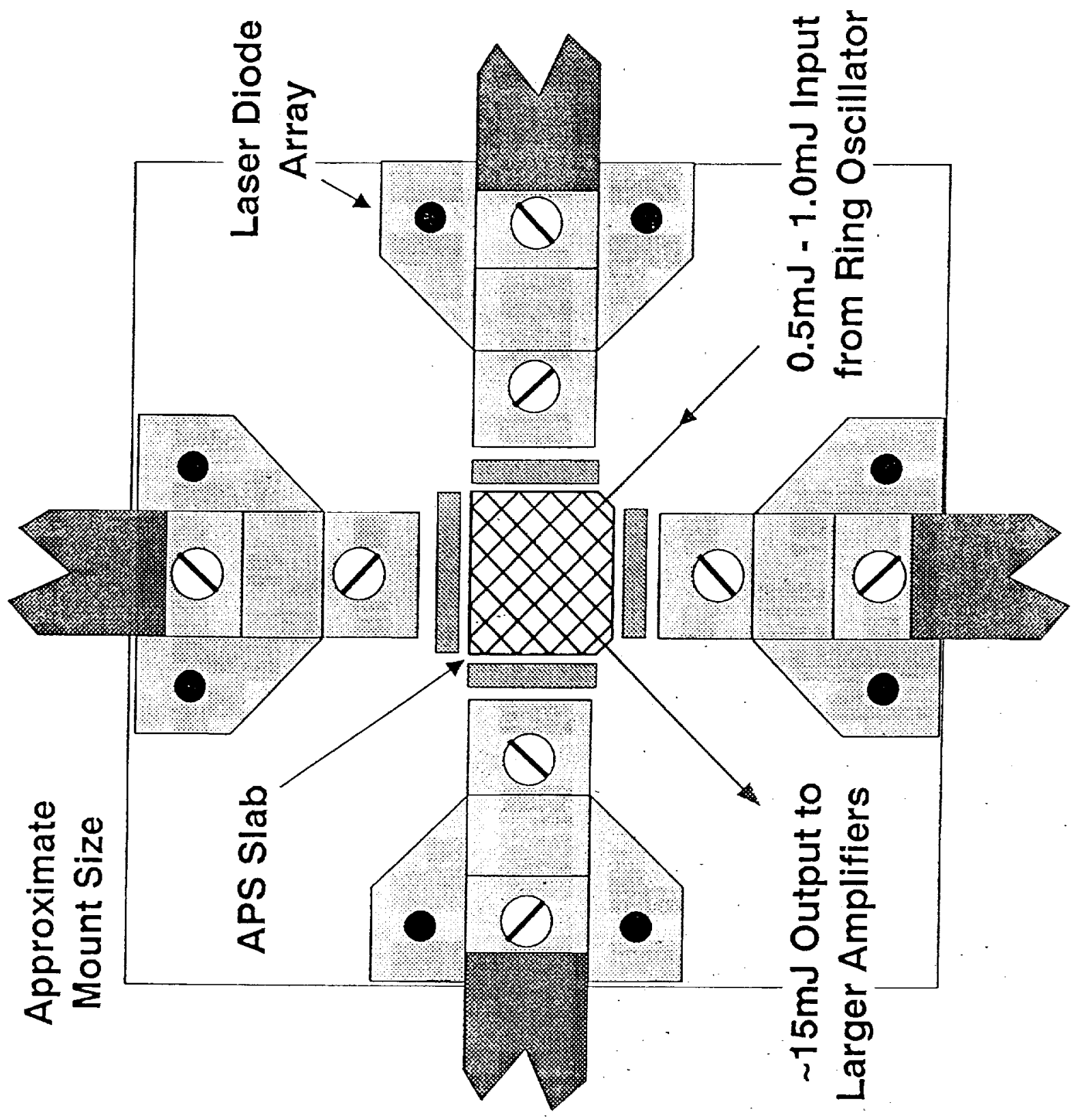

